Editorial

\title{
Reparación Ligamento Cruzado Anterior, ¿Mito o Realidad?
}

\section{Anterior Cruciate Ligament Repair, ¿Myth or Truth?}

\author{
Sergio Arellano Garrido ${ }^{1,2}$ \\ ${ }^{1}$ Clínica Alemana de Santiago, Santiago, Chile \\ 2 Hospital Padre Hurtado, San Ramón, Región Metropolitana, Chile \\ Rev Chil Ortop Traumatol 2019;60:77-78.
}

La rotura del ligamento cruzado anterior (LCA) es una de las lesiones de rodilla más frecuentes. ${ }^{1}$ Sabemos que su importancia radica en que es el responsable de la restricción del $85 \%$ de la traslación anterior de la tibia sobre el fémur. Diversos estudios han demostrado que su ausencia, conlleva un mayor riesgo de lesiones condrales y meniscales con el paso del tiempo. ${ }^{2}$ Sin embargo, no está del todo claro que tenga un rol directo en la presencia de artrosis, si bien suena una secuencia lógica.

Hasta los años 90 el tratamiento de las lesiones de LCA era con reparación abierta, lo cual conllevaba una gran morbilidad post operatoria con pobres resultados a corto plazo. ${ }^{3}$

Con la aparición de la artroscopía, la reconstrucción fue consolidándose hasta ser hoy el "gold standard" del tratamiento en lesiones de LCA. Esta técnica ha demostrado resultados reproducibles con un retorno deportivo $>80 \%$, buenos y excelentes resultados funcionales en $>85 \%$ de los pacientes, tasas de falla de un $2-5 \%$, y acorde al último estudio de Nwachukwu en el JBJS 2017, un $98 \%$ de los pacientes volvería a operarse. ${ }^{4}$

Entonces ¿por qué cambiar?

Entre las ventajas que se proponen de la reparación LCA vs la reconstrucción destacan:

- Preservación fibras nativas LCA (propiocepción)

- Evita morbilidad de sitio donante (injerto), menos invasivo

- En caso de falla, se tiene la opción de la reconstrucción

Como bien dijo Erick Hohmann en una editorial de la Arthroscopy en 2016, "el fin último de cualquier intervención en medicina es restaurar la condición previa.. ${ }^{5}$ Basándonos en este concepto, la reparación del LCA suena lo más lógico para lograr este objetivo. Es por ello que en los últimos 5 años la cantidad de estudios publicados sobre reparación del LCA han aumentado exponencialmente, confirmando el gran interés en torno a este tema. Acompañado de una intervención importante de la industria, creando sistemas acordes para lograr esta reparación de mejor manera. Las revisiones sistemáticas mostraban hasta el año 2017 tasas de falla de alrededor de un $20 \%$ y retorno deportivo $<65 \%$, con lo cual se estaba muy lejos de equiparar los resultados obtenidos con la reconstrucción. ${ }^{6}$ Sin embargo en los últimos años se logró identificar el factor clave para lograr reparaciones exitosas, que es la selección adecuada del paciente. No todos los pacientes son candidatos a una reparación.

Como lo demostró Van der List en su publicación en The Knee 2017, solo se logran resultados comparables a la reconstrucción cuando se selecciona adecuadamente al paciente, siendo los candidatos ideales ${ }^{7}$ :

- Lesiones proximales de LCA (Sherman I-II)

- Remanente de LCA de buena calidad (tejido)

- Lesiones agudas (<3 semanas)

Sumado a las características idóneas del paciente para optar por la reparación, está también la técnica quirúrgica, donde el uso del internal brace como protección de la reparación ha demostrado una menor tasa de falla en los últimos estudios. ${ }^{8}$

Hace algunos meses se publicó una revisión sistemática realizada por Nwachukwu en Arthroscopy, reunió más de 2.400 paciente en 28 estudios. Considerando desde el año 2003-2018. Demostró resultados funcionales comparables a la reconstrucción (Lysholm e IKDC), retorno deportivo precoz comparado con reconstrucción (14 semanas), satisfacción de los pacientes $90 \%$, pero con tasas muy altas aún de re-rotura y revisión ( 23 y $12 \%$ respectivamente). ${ }^{9}$ Esto probablemente en contexto que abarca estudios desde años iniciales donde no se consideraba aun las características que debía tener el paciente para ser el candidato ideal a optar por esta cirugía.

La reparación del LCA está emergiendo como una alternativa válida, sin embargo, aún no contamos con estudios prospectivos ni de seguimiento a largo plazo que nos permitan incentivar y avalar del todo el uso de esta técnica. Claramente enfrentamos nuevamente un cambio de paradigma frente al tratamiento de las lesiones de LCA, pero solo el tiempo nos permitirá saber si se mantiene en el futuro o será nuevamente desestimado como lo fue con la aparición de la artroscopía hace casi 30 años.

Copyright (e) 2019 by Thieme Revinter Publicações Ltda, Rio de Janeiro, Brazil
Address for correspondence Sergio Arellano G., MD, Clínica Alemana de Santiago, Av. Vitacura 5951, Vitacura, Santiago, Chile (e-mail: sarellano@alemana.cl).
DOI https://doi.org/ 10.1055/s-0039-3400287. ISSN 0716-4548.

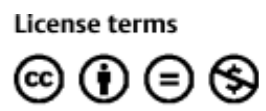


Lo que la literatura nos permite pensar actualmente, es que en la medida que seamos capaces de identificar al paciente ideal para este procedimiento, nuestras chances de éxito aumentan considerablemente.

\section{Conflicto de Intereses}

Lo autor del trabajo declara no tener ningún conflicto de intereses.

\section{References}

1 Sanders TL, Maradit Kremers H, Bryan AJ, et al. Incidence of anterior cruciate ligament tears and reconstruction: A 21-year populationbased study. Am J Sports Med 2016;44(06):1502-1507

2 Demey G, Magnussen RA, Lustig S, Servien E, Neyret P. Total knee arthroplasty for advanced osteoarthritis in the anterior cruciate ligament deficient knee. Int Orthop 2012;36(03):559-564

3 Kaplan N, Wickiewicz TL, Warren RF. Primary surgical treatment of anterior cruciate ligament ruptures. A long-term follow-up study. Am J Sports Med 1990;18(04):354-358
4 Nwachukwu BU, Voleti PB, Berkanish P, et al. Return to play and patient satisfaction after ACL reconstruction: Study with minimum 2-year follow-up. J Bone Joint Surg Am 2017;99(09): 720-725

5 Hohmann E. Editorial Commentary: Renaissance of Primary Anterior Cruciate Ligament Repair: Is History Repeating Itself? Arthroscopy 2016;32(12):2570-2571

6 van der List JP, DiFelice GS. Role of tear location on outcomes of open primary repair of the anterior cruciate ligament: A systematic review of historical studies. Knee 2017;24(05):898-908

7 van der List JP, DiFelice GS. Range of motion and complications following primary repair versus reconstruction of the anterior cruciate ligament. Knee 2017;24(04):798-807

8 Jonkergouw A, van der List JP, DiFelice GS. Arthroscopic primary repair of proximal anterior cruciate ligament tears: outcomes of the first 56 consecutive patients and the role of additional internal bracing. Knee Surg Sports Traumatol Arthrosc 2019;27 (01):21-28

9 Nwachukwu BU, Patel BH, Lu Y, Allen AA, Williams RJ III. Anterior Cruciate Ligament Repair Outcomes: An Updated Systematic Review of Recent Literature. Arthroscopy 2019;35(07):2233-2247 